\title{
Aula virtual para el aprendizaje del proceso de diseño arquitectónico
}

\author{
Virtual classroom for learning the architectural design process
}

iD Mayre Molina Zambrano; mayremolina@gmail.com

iD Yovanni Alexander Ruiz Morales; yruizmorales@gmail.com

Universidad Nacional Experimental del Táchira (Venezuela)

\section{Resumen}

Se planteó el diseño de un aula virtual para el aprendizaje del proceso del diseño arquitectónico basado en tres aspectos fundamentales: 1) perfil de los estudiantes (estilos de aprendizaje, competencias en el diseño arquitectónico y habilidades digitales), 2) diseño instruccional, y 3) valoración del diseño instruccional una vez construida el aula. La investigación fue de carácter cuantitativo, alcance descriptivo y diseño no experimental. La muestra intencional correspondió a 110 estudiantes de arquitectura inscritos en la asignatura Proyectos III, lapso académico 2019-3, y 5 expertos en: diseño instruccional, currículo y evaluación. Los resultados obtenidos fueron: 1) perfil de los estudiantes, A) estilos de aprendizaje, Sensitivo (63\%) Intuitivo (37\%), Visual (97\%) Verbal (3\%), Activo (71\%) Reflexivo (29\%), y Secuencial (66\%) Global (34\%); B) nivel de habilidades y destrezas digitales, Alto (78,70\%), Medio (18\%) y Bajo (3,30\%); y C) competencias y habilidades en el proceso de diseño arquitectónico, conceptuales $(19,8 \%)$ y procedimentales (24\%). 2) El diseño instruccional se basó en Merril (2012), ADDIE, y Dick y Carey. 3) los hallazgos de la evaluación del diseño instruccional indicaron que el aula virtual reúne calidad en: claridad de la información (93\%), práctica reflexiva (90\%), realimentación informativa (73\%) y motivación de los estudiantes (100\%).

Palabras clave:

Aula virtual; diseño de aula; diseño instruccional; enseñanza en arquitectura; enseñanza superior.

\begin{abstract}
The design of a virtual classroom was planned for architectural design process learning based on three fundamental aspects: (1) profile students' (learning styles, architectural design competencies and digital skills), (2) instructional design, y (3) assessment of instructional design once the classroom is built. The research was quantitative, descriptive in scope and non-experimental. The intentional samples corresponded to 110 architecture students enrolled in Projects III, academic period 2019-3 and 5 experts in: instructional design, evaluation and curriculum. The results obtained were: (1) profile students', A) learning styles, Sensitive (63\%) Intuitive (37\%), Visual (97\%) Verbal (3\%), Active (71\%) Reflective (29\%), Sequential (66\%) Global (34\%); B) Digital skills and skills level, High (78.70\%), Medium (18.00\%) and Low (3.30\%); and C) Competencies and skills in the architectural design process, conceptuals (80.2\%) and procedurals (24\%). (2) The Instructional design was based on Merril (2012), ADDIE, and Dick and Carey. (3) The findings of the instructional design assessment indicated that the virtual classroom brings together quality in: clarity of information (93\%), promotes thoughtful practice (90\%), informative feedback (73\%) and promotes student motivation (100\%).
\end{abstract}

\section{Keywords:}

Virtual classrooms; classroom design; instructional design; architectural education; higher education. 


\section{INTRODUCCIÓN}

La enseñanza del diseño arquitectónico se basa en procesos de modelos creativos que van desde la concepción hasta la producción física de resultados, razón por la cual se ha apoyado de métodos orientados a la solución de problemas y el aprender haciendo propuesto por Schön (1983), el modelo de aprendizaje experiencial de Kolb (2015) y el Blended Learning. En cuanto a las experiencias con E-learning, las investigaciones orientadas al diseño e implementación de cursos para la enseñanza del diseño arquitectónico son escasos (Fleishman, 2018), y en el uso del Blended Learning, los estudios de Fleischmann (2018), Masdéu y Fuses (2017) y Alkiser (2017) coinciden con Fleischmann (2020) al afirmar que durante el proceso de aprendizaje del diseño arquitectónico se debe mantener la retroalimentación continua brindada por el docente, apoyada del conocimiento, de la reflexión en acción, y de la noción al ejecutar el proceso de diseño por parte del estudiante, situación que en un aprendizaje virtual se dificultaría.

Otros estudios (Hill, 2017; Joklová y Pifko, 2015; Lotz et al., 2015) demostraron que la fusión adecuada de las prácticas de enseñanza del taller de diseño y la adaptación de metodologías de aprendizaje virtual enfocadas en tareas, ofrecen resultados favorables. Hill (2017) y Joklová y Pifko (2015) realizaron sus investigaciones para la enseñanza del proceso de diseño arquitectónico en estudiantes universitarios, se enfocaron en el uso de materiales instruccionales, asignación de tareas relacionadas con la metodología del proceso proyectual, retroalimentación del docente a las propuestas de los estudiantes y evaluación entre pares. Los hallazgos obtenidos destacan que esta metodología posibilita el desarrollo de habilidades para el proceso proyectual.

En esta misma línea de trabajo con Entorno Virtual de Aprendizaje (EVA), Lotz et al. (2015) analizaron el nivel de compromiso de los estudiantes que participaron en un Entorno Virtual de Aprendizaje (EVA), cuyo objetivo fue el diseño de un proyecto arquitectónico, para ello se implementaron actividades de aprendizaje contextualizadas, seguimiento por parte del docente a la ejecución proyectual y evaluación formativa de los estudiantes a los avances del proyecto. Los resultados indican que la propuesta de tareas situadas y la participación activa de los estudiantes como agentes evaluadores, promueve el compromiso, un alto nivel de participación y motivación para el aprendizaje de la representación y conceptualización del proceso proyectual.

Estas prácticas permiten inferir que los EVA para la enseñanza del diseño arquitectónico deben articular adecuadamente la tecnología con estrategias didácticas que contemplen la realización de tareas, el uso de medios, herramientas y recursos digitales destinados a la transformación cognitiva del estudiante (Ruiz-Morales et al., 2017).

En este sentido, el soporte de un aula mediada por herramientas tecnológicas es el Diseño Instruccional (DI). Según Prado-Rodríguez (2021) el DI tiene como propósito orientar el trabajo didáctico del docente en las etapas de planificación, desarrollo de los medios y diseño de estrategias de enseñanza y aprendizaje. 
Frente a este panorama de incorporación de las Tecnologías de Información y Comunicación (TIC) para la enseñanza del diseño arquitectónico, el presente estudio plantea diseñar un entorno virtual para el aprendizaje del proceso del diseño arquitectónico basado en los principios instruccionales de Merril y el modelo ADDIE, que considere el perfil de los estudiantes (estilos de aprendizaje, competencias en el diseño arquitectónico y habilidades digitales), y valore la calidad del diseño instruccional una vez producida el aula. Se plantea explorar el perfil de los estudiantes para conocer: 1) la forma como aprenden los estudiantes, 2) las habilidades y destrezas ya adquiridas del contenido disciplinar a enseñar, y 3) capacidades en el manejo de información, comunicación, tecnología y organización.

\subsection{El proceso de diseño arquitectónico}

El proceso de diseño arquitectónico se refiere a un conjunto de procedimientos para la concreción física de un objeto, transformando un propósito en unas condiciones espaciales nuevas donde se desenvolverá el ser humano, y requiere un "sistema de pensamiento y actuación orientado a satisfacer necesidades humanas mediante la creación de una intención y su transformación de una realidad material futura" (Guevara, 2013, p. 119).

En cuanto a la actuación requerida durante el proceso de diseño arquitectónico: Salama (2015) indica la integración de la capacidad lógico/racional y la intuitiva/imaginativa, por su parte, Bashier (2014) introduce la teoría racional de solución de problemas y la reflexión en acción, cuyas actividades resultantes derivan de un proceso holístico. De acuerdo a ello, Reiter et al. (2009) refieren la importancia de aprender el proceso de diseño arquitectónico mediante modelos de procesos creativos, que requieran del estudiante poner en práctica habilidades cognitivas de atención, comprensión, análisis y elaboración.

Respecto a los modelos de procesos creativos para el aprendizaje proyectual, Mattingly (2011) realizó una revisión sistemática de literatura producida entre el año 1997 y 2009, en la cual encontró tres categorías en común: análisis, conceptualización y síntesis. Diversas investigaciones (Soliman, 2017 ; Opoko et al., 2015; Basier, 2014) valoran el Método de Diseño de Cantú (1998) como un modelo creativo, compuesto por la siguiente estructura: 1) Formulación, comprende: planteamiento del problema, análisis del marco referencial y del estudio de caso; 2) Investigación y análisis de datos obtenidos del medio físico, usuario, y aspecto legal y normativo; 3) Procesamiento, se generan insumos como: programa de áreas, diagramas de relaciones, objetivos y criterios de diseño; 4) Síntesis, se plantea la zonificación y la conceptualización integrando aspectos contextuales, funcionales, formales, tecnológicos y de significado; y 5) Diseño y Evaluación, se produce el anteproyecto y el proyecto.

\subsection{El Diseño Instruccional (DI)}

El diseño instruccional (DI) es un proceso sistemático que traduce los principios generales de aprendizaje e instrucción, para desarrollar materiales educativos y el entorno, buscando crear cambios en los conocimientos y habilidades del estudiante (Khalil y Elkhider, 2016). Su proceso exige: delimitación adecuada de los objetivos, selección rigurosa de actividades, recursos, y una secuencia apropiada de contenidos para lograr el alcance de las metas educativas (López y Peña, 2019), mediante la selección de teorías de aprendizaje, modelos pedagógicos y 
elementos didácticos específicos alineados con el contenido disciplinar a enseñar (Candela, 2016).

La conceptualización del DI se basa en: 1) las teorías de aprendizaje, explican la manera como se produce el aprendizaje en el interior del estudiante; 2) las teorías de la enseñanza, especifican las condiciones para optimizar el aprendizaje (Khalil y Elkhider, 2016); y 3) teorías psicológicas, pedagógicas y sociológicas que intervienen en corrientes como: conductismo (enfocado en la consolidación de la respuesta), cognitivismo (basado en la adquisición del conocimiento), constructivismo (sustentado en la elaboración del conocimiento), y conectivismo (caracterizado por el aprendizaje en redes) (Prado-Rodríguez, 2021). Estas proposiciones han influido en modelos de DI para sistematizar el desarrollo de acciones formativas, destacando: el de Gadné, ASSURE, Jonassen, ADDIE y Dick y Carey.

En los entornos de enseñanza virtuales, la reformulación de los DI vincula la dimensión tecnológica y pedagógica cuyo proceso amerita un pensamiento múltiple, dialéctico, holístico y flexible (Dick et al. 2015). Su enfoque configura la acción formativa centrada en el estudiante y en el aprendizaje como resultado de la influencia de las teorías de aprendizaje pre-existentes, el reconocimiento del contexto, las características de los estudiantes, el papel de la motivación y las herramientas digitales (López y Peña, 2019). Razón por la cual, el uso de modelos de DI guían y sistematizan el desarrollo de las acciones formativas como garantía de validez en todo el proceso.

\subsection{Principios fundamentales del diseño de la instrucción}

Los principios fundamentales de la instrucción de Merril (2012) proporcionan las maneras de proceder para realizar las actividades que apoyarán el aprendizaje. Estos se describen a continuación:

- Situación problema: se plantea las instrucciones, condiciones y requisitos para resolver un problema o fenómeno de estudio.

- Activación: consiste en recurrir a experiencias previas que permitan la organización del nuevo conocimiento.

- Demostración: se presenta el contenido a los estudiantes con medios apropiados para mostrar habilidades (de qué clase es, cómo se hace y qué sucede en la ejecución) que contribuyen a solucionar el problema.

- Aplicación: refiere la utilización del conocimiento para la resolución de problemas, en el marco de un proceso proyectual caracterizado por la mediación docente y la retroalimetación.

Integración: contempla la articulación entre el análisis, síntesis y diseño; lo cual requiere la indagación de datos, formulación de ideas y evaluación de estas mediante un proceso reflexivo basado en la acción proyectual. 


\subsection{Modelos de diseño instruccional}

Los modelos de diseño instruccional utilizados en el diseño del aula virtual fueron ADDIE, y el Modelo de Dick y Carey, estos se describen a continuación:

ADDIE comprende las siguientes fases definidas por Khalil y Elkhider (2016): a) Análisis se identifican las características de los estudiantes y el contexto, se determinan las metas de la instrucción y el entorno de aprendizaje; b) Diseño, se establecen los objetivos de aprendizaje para delinear el contenido y las estrategias de instrucción. También, se seleccionan los tipos de actividades de aprendizaje, los recursos y formas de evaluación; c) Desarrollo, se crean y producen los contenidos instruccionales, el prototipo del aula y los instrumentos de evaluación; d) Implementación, incluye publicación de los materiales de instrucción para fomentar el dominio de los objetivos de aprendizaje por parte de los estudiantes; y e) Evaluación, valora procedimientos y actividades realizadas antes de la implementación (evaluación formativa), y luego de la implementación (evaluación sumativa).

Modelo de Dick y Carey en sus etapas considera el diagnóstico de necesidades y su contexto, mediante fases como: a) Identificar metas educativas; b) Realizar análisis instruccionales; c) Identificar los comportamientos de entrada y las características del alumno; d) Definir los objetivos de desempeño; e) Desarrollar instrumentos de evaluación; f) Desarrollar la estrategia instruccional; g) Desarrollar y seleccionar los materiales instruccionales; y h) Desarrollar y realizar la evaluación formativa y sumativa (Dick et al., 2015).

\section{MÉTODO}

El estudio planteó el diseño de un aula virtual para el aprendizaje del proceso del diseño arquitectónico. Se consideró: 1) perfil de los estudiantes (estilos de aprendizaje, competencias en el diseño arquitectónico y habilidades digitales), 2) diseño instruccional, y 3) valoración del diseño instruccional una vez producida el aula.

La investigación fue de carácter cuantitativo, de alcance descriptivo y diseño no experimental. Las muestras de tipo intencionado correspondieron a 110 estudiantes de Proyectos III (lapso académico 2019-3) de la carrera de arquitectura de la Universidad Nacional Experimental del Táchira (UNET), Venezuela, y 5 expertos en: diseño instruccional (2), currículo y evaluación (3). Los instrumentos utilizados fueron:

1) Index of Learning Styles de Felder y Soloman (1997), la cual reportó una Confiabilidad por re- aplicación de pruebas de 0,82.

2) Rúbrica para explorar las competencias y habilidades en el proceso de diseño arquitectónico basado en Cantú (1998).

3) Cuestionario sobre habilidades digitales de Organista et al., (2016), con Coeficiente de Validez de Contenido (CVC) de 0,95 y confiabilidad 0,81.

4) Inventario del diseño instruccional en cursos de formación por Coronado y Barraza (2018), con CVC de 0,90. 


\section{RESULTADOS}

\subsection{Perfil de los aspirantes del curso}

Información general: Sexo femenino (64\%) y masculino (36\%). Edad entre los 19 y 22 años.

Preferencias de estilos de aprendizaje: a) Tipo de información a percibir, Sensitivo (63\%) Intuitivo (37\%); b) Vía sensorial para captar la información, Visual (97\%) Verbal (3\%); c) Forma de adquirir la información. Activo (71\%) Reflexivo (29\%); d) Modo para el entendimiento de contenidos, Secuencial (66\%) Global (34\%).

Las competencias y habilidades en el proceso de diseño arquitectónico adquiridas por los estudiantes, referidas a lo conceptual y procedimental de los métodos utilizados en la formulación, investigación, procesamiento, síntesis y diseño, los resultados indicaron que: la mayoría conoce la definición de estos al señalar el nivel De Acuerdo $(80,2 \%)$ y Desacuerdo $(19,8 \%)$ en las afirmaciones presentadas. En cuanto al uso de los métodos en la fase de prediseño manifestaron Dificultad (76\%) y Facilidad (24\%).

Nivel de habilidades y destrezas digitales orientadas a la actividad educativa para el manejo de: información (87,80\%), comunicación (82,9\%), tecnología (61,43\%), y aspectos de organización $(82,45 \%)$. En promedio el $78,70 \%$ se percibe con una Alta competencia digital y el $21,30 \%$ manifiesta Dificultad. Las actividades de Proyectos arquitectónicos las realizan con medios análogos (100\%) y digitales (16\% promedio) y poseen: teléfono inteligente (85\%), computadora de escritorio (83\%), laptop (40\%) y Tableta (26\%).

\subsection{Diseño del aula virtual}

En la construcción del aula virtual se empleó el modelo ADDIE, según Khalil y Elkhider (2016) proporciona un proceso sistemático y flexible para diseñar y desarrollar una experiencia de aprendizaje, cuyas fases se pueden elaborar detalladamente con el modelo de Dick y Carey.

\subsubsection{Análisis}

El contexto de la institución anfitriona, la UNET, posee infraestructura tecnológica, administrativa y gestión académica curricular para la implementación, evaluación y mantenimiento del aula virtual soportada en la plataforma MOODLE. El contenido a desarrollar del curso se basó en la enseñanza de la metodología del proceso de diseño arquitectónico según la unidad curricular de Proyectos III (Universidad Nacional Experimental del Táchira, 2007) y Cantú (1998).

A partir de los hallazgos obtenidos del perfil de los estudiantes se estableció: 1) Uso de estrategias didácticas según la participación del estudiante, estrategias para el aprendizaje significativo y la abstracción; 2) Materiales instruccionales constituidos por recursos con buena presentación visual, animación multimedia y Objetos de Aprendizaje; 3) Selección de medios tecnológicos adecuados a las estrategias de enseñanza aprendizaje planteadas para la enseñanza de contenido y de uso común para los estudiantes; y 4) Entrenamiento de 
habilidades procedimentales para el desarrollo de la fase de pre-diseño (formulación e investigación y procesamiento) del proceso de diseño arquitectónico.

Los conocimientos y necesidades de aprendizaje obtenidos de la rúbrica para explorar las competencias y habilidades en el proceso de diseño arquitectónico de los estudiantes se definieron mediante la lista de metas planteadas por Dick et al., (2015) como: Formular el problema de estudio; Analizar la información específica y normativa del lugar; Elaborar el programa arquitectónico; Estudiar las relaciones funcionales; Zonificar la propuesta arquitectónica; Definir el concepto generador; y Diseñar la propuesta arquitectónica. Posteriormente, se estableció los niveles de competencias de entrada y de salida fundamentadas en los nuevos conocimientos a construir en los estudiantes.

Se utilizó la instrucción centrada en tareas y la metodología del aprendizaje basado en problemas para el entrenamiento de competencias genéricas y específicas, el pensamiento crítico y el aprendizaje autorregulado. El problema a resolver corresponde a la agrupación de espacios abiertos y cerrados (desarrollo de conjunto) definido como: propuesta arquitectónica de una instalación de actividades recreativas y deportivas en una superficie de 8.668,45 m2 como respuesta a las necesidades de una zona urbana de uso residencial.

De acuerdo con Merril (2012) la búsqueda de soluciones al problema formulado se realiza por fases para el logro de una comprensión gradual de los estudiantes, también se plantean situaciones de enseñanza orientadas al desarrollo de modos de pensamiento para la toma de decisiones basadas en el ciclo de Kolb (2015): experimentación activa, experiencia concreta, observación reflexiva y abstracción, lo que exigió la integración de estrategias que favorezcan el diálogo reflexivo, para Webster (2004) se evidencian mediante la revisión y reflexión constante sobre el trabajo realizado y el logro de los objetivos pedagógicos esperados, con énfasis en el desarrollo meta-cognitivo, la articulación de retroalimentación, la construcción de conocimiento colectivo y del significado.

En el caso de la retroalimentación, se seleccionaron estrategias como: 1) Elaboración de tareas para la solución de problemas; 2) Evaluación por pares, con participación activa y pasiva en el aprendizaje, apoyadas con rúbricas y escalas; 3) Uso del E-Portafolio para permitir conexiones significativas entre el contenido y experiencia práctica. En la fase de pre-diseño, las actividades se fundamentaron en el aprendizaje colaborativo para construir y aumentar la validez de las ideas.

\subsubsection{Diseño}

El objetivo general del curso se definió así: desarrollar habilidades y destrezas en la metodología del proceso de diseño arquitectónico. Los objetivos específicos se describen a continuación:

1. Formular el problema de estudio para analizar las circunstancias que lo afectan mediante la investigación e identificación de las variables y determinantes.

2. Analizar el lugar para caracterizar los factores físicos, construidos, sociales, económicos y normativos interpretándolos, comprendiéndolos y adecuándolos a la propuesta.

3. Obtener las áreas que constituyen el objeto arquitectónico para determinar la magnitud del espacio a través del análisis de las necesidades del usuario. 
4. Relacionar las áreas propuestas del objeto arquitectónico mediante el estudio de vínculos funcionales, proximidad, importancia, uso y acceso.

5. Elaborar un diagrama general con áreas proporcionadas y relacionadas, implantadas en el terreno tomando en cuenta las opciones de desarrollo formuladas.

6. Definir alternativas de solución de la propuesta arquitectónica de forma coherente y estructurada en aspectos contextuales, funcionales, formales, tecnológicos y de significado.

7. Desarrollar los esquemas preliminares de la propuesta arquitectónica.

El contenido del curso se estructuró según cada objetivo específico y estos constituyeron siete unidades didácticas la cual, se les definió la competencia específica y el nivel de conocimiento: conceptual, procedimental y actitudinal. Las estrategias instruccionales correspondieron a estrategias didácticas (métodos, técnicas y actividades) para promover el aprendizaje constructivo: pensamiento crítico, creatividad, resolución de problemas y trabajo colaborativo mediante actividades de experimentación y elaboración, fundamentadas en la instrucción basada en tareas a través de: a) Interacción síncrona y asíncrona, apoyada de Foros y Chat; b) Uso de wikis; c) Elaboración de productos: fichas, tablas, mapas, diagramas, gráficos, y videos para evidenciar aprendizajes, realizar co-evaluación y construir tareas posteriores; d) WebQuest o investigación dirigida; e) Guía didáctica para el seguimiento autónomo del curso; y f) Feedback en la evaluación formativa, sumativa y final.

A cada unidad didáctica se le realizó: 1) Matriz integradora de los componentes didácticos: conocimientos (conceptuales, procedimentales y actitudinales) y la estrategia instruccional, donde se establecieron 21 actividades de aprendizaje con su respectivo medio tecnológico; 2) Matriz de recursos didácticos (recursos básicos, a profundizar y otros medios); 3) Cronograma de actividades (duración: 16 semanas); y 4) Matriz de evaluación (actividad de aprendizaje, criterios de evaluación, evidencia, instrumento y ponderación).

Entre los recursos didácticos, se plantearon cinco Objetos de Aprendizaje (OA) para las primeras unidades didácticas correspondientes a la fase de pre-diseño, estos se constituyeron de la metodología WebQuest (estructurada en tareas, medios, herramientas y recursos).

El proceso de evaluación de las actividades contempla:

- Evaluación Diagnóstica, uso de Rúbrica

- Evaluación Formativa, incluye la participación del estudiante en el proceso de valoración mediante la observación sistematizada con el uso de escalas y rúbricas aplicada a ocho actividades (foros, cuadros sinópticos, mapas cognitivos, fichas y tablas), y la muestra de desempeño del estudiante se evidenciará con el E-portafolio. Durante la implementación del aula se realizará el seguimiento de la frecuencia de interacción del estudiante en Foros y Chats.

- Evaluación Sumativa, se empleará la observación sistematizada y el análisis de diferentes productos con el uso de rúbricas para valorar catorce actividades (informes, gráficos, presentaciones, fichas y productos). 


\subsubsection{Desarrollo}

Comprendió la construcción de: los recursos instruccionales de aprendizaje y de enseñanza, el aula virtual y los instrumentos de evaluación. Estos se especifican a continuación:

Recursos instruccionales: se basaron en estrategias didácticas: 1) Pre-instruccionales, activan conocimientos y experiencias previas (organizadores previos); 2) Co-instruccionales, logran una mejor codificación y conceptualización del contenido (mapas conceptuales, esquemas e infografías); y 3) Post-instruccionales, ofrecen una visión sintética, integradora y crítica del material (resumen final y mapas conceptuales).

El material instruccional desarrollado comprendió la siguiente estructura: 1) Definición de la unidad didáctica basada en las necesidades a enseñarse; 2) Competencia a desarrollar; 3) Estrategias pedagógicas y didácticas: aprendizaje guiado y colaborativo para la síntesis de información; 4) Actividades de: comprensión, análisis, y síntesis de la información; 5) Técnica: instrucción programada (WebQuest) y trabajo colaborativo; y 6) Medio didáctico: cinco objetos de aprendizaje.

El desarrollo del guion didáctico correspondiente a cada unidad de contenido se constituyó de: 1) Propósito; 2) Estructura: subunidades didácticas, competencia general y plan de evaluación; 3) Presentación: definición, finalidad, competencias a adquirir, desarrollo, evaluación y duración; 4) Desarrollo de cada tema según las competencias específicas estructurado en: Tarea, Proceso a seguir, Recursos y Evaluación; 5) Evaluación; y 6) Bibliografía.

La guía didáctica basada en Aguilar (2004) se organizó en: 1) Portada (identificación de la unidad curricular, institución y autores); 2) Índice de contenidos; y 3) introducción. El desarrollo se estructuró así: a) Aspectos generales como: justificación, propósito, grupo de incidencia, competencias y pre-requisitos; b) Proceso de aprendizaje, especifica: objetivos, recursos y medios, contenidos de la unidad didáctica, competencias de las sub-unidades didácticas, bibliografía, y orientaciones generales (presentación del docente, descripción de los recursos, instrucciones de uso de la guía y del aula, y cronograma de actividades). El apartado de Actividades muestra: unidad didáctica, introducción, objetivos, estrategias de aprendizaje, proceso a seguir, y evaluación de los aprendizajes (actividad, evidencia, instrumento, modalidad de participación y ponderación).

Objetos de Aprendizaje (OA): su construcción se basó en la metodología de Dorrego (1994) con fases de producción, realización y evaluación apoyadas de: guion de contenido, didáctico y técnico. El contenido de los OA se organizó según Molina-Zambrano y Ruiz-Morales (2020) en: 1) Introducción, describe la importancia del tema y los objetivos; 2) Tarea, detalla las actividades de aprendizaje; 3) Proceso, instruye la realización de las actividades; 4) Recursos, presenta los materiales digitales en diversos formatos; y 5) Evaluación, especifica la participación del estudiante, la ponderación y proporciona el instrumento.

Videos didácticos: para la instrucción se planteó el desarrollo de tres videos cuya intención didáctica fue: Introducir al tema, capacitar en el manejo de metodologías para la formulación del problema (unidad I), ofrecer orientación procedimental, estudio y análisis de la topografía (unidad II) y estrategias para realizar la zonificación (unidad V) (Molina-Zambrano, 2018). La 
construcción de los videos se elaboró en tres fases según (Vélez, 2017): pre-producción, producción, y postproducción.

El aula virtual se construyó en MOODLE, se organizó según el instructivo de la Coordinación de Educación a Distancia (2016) para el desarrollo del aula virtual en: 1) Zona informativa, agrupa recursos como: video de bienvenida y foro, guía didáctica, programa analítico, presentación de los docentes, infografía del curso, orientaciones generales del uso del aula y blog de consulta; 2) Biblioteca; 3) Zona de comunicación; 4) Zonas de progreso, corresponden al contenido de cada unidad didáctica, incluye: Sala de estudio (organizador previo, OA y demás recursos) y Actividades (presentación, requerimientos de entrega, criterios de evaluación y duración); y 5) Zona de clausura: instrumento de evaluación del diseño instruccional.

Instrumentos de evaluación: para la valoración de las 21 actividades se seleccionaron rúbricas y escalas, los criterios se especifican a continuación:

1. Presentaciones, fichas y diagramas (rúbrica): a) contenido: dominio y comprensión, planificación y organización, integración; b) aplicación práctica: coherencia con la metodología sugerida; y c) apoyo visual: cantidad de información y explicación, legibilidad, relevancia y adecuación gráfica (García, 2011).

2. Informes (rúbrica): formato y presentación, organización, referencias bibliográficas, autoría, logro del trabajo (Mora et al., 2018).

3. Foros (rúbrica): a) conocimientos, vocabulario y sintaxis; b) construcción del argumento, postura, estructura, transiciones, justificación y contra argumentación; c) actitudes, crítica, respetuosa y de colaboración (Guzmán et al., 2012).

4. Mapas cognitivos (escala): modalidad del lenguaje, fases del acto mental, input, elaboración, output, nivel de complejidad, de abstracción, eficacia (Avendaño y Parada, 2012).

5. Productos de la propuesta arquitectónica, escala comparativa del nivel de creatividad: original/poco original, bien desarrollado/falta de desarrollo, sorprendente/esperado, ordenado/desordenado, asombroso/convencional, funcional/no funcional, único/repetitivo y lógico/ilógico (Oman et al., 2013).

6. E-portafolio (rúbrica): a) estructura, presentación, desarrollo y conclusión; b) elementos de evidencia, calidad de los productos surgidos, y proceso reflexivo; y aspectos transversales, competencia comunicativa, manejo de la tecnología (Tur y Urbina, 2016).

7. Inventario del diseño instruccional (escala): claridad de la información, práctica reflexiva, realimentación informativa y motivación intrínseca y extrínseca (Coronado y Barraza, 2018).

Posteriormente, se ejecutaron los guiones diseñados para la realización de los OA, los videos, el material instruccional y el entorno virtual en plataforma MOODLE (Figura 1 y 2). 


\section{Figura 1}

Captura de pantalla del aula virtual: Zona informativa y zona de comunicación

\section{U N E T Virtual}

n Página Principal $>M i>P o>M ~>A u$

\section{Proyectos III}

Libro: El Arte de Proyectar en Arquitectura
Libro: Arquitectura, forma y orden
Libro: Manual de conceptos y formas arquitectónicas
Libro: Sistemas de Estructuras
Libro: Discapacidad y Diseño Accesible

ZONA DE COMUNICACIÓN

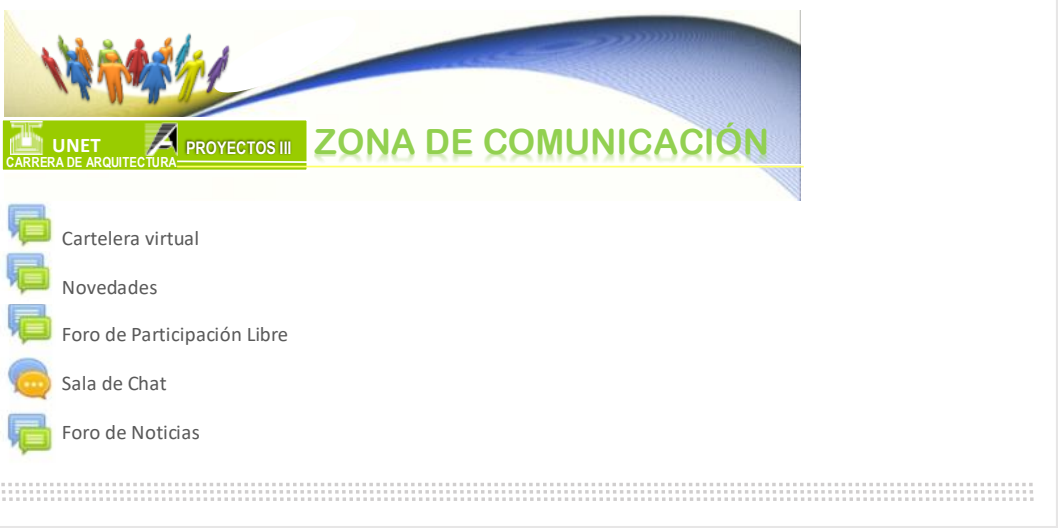




\section{Figura 2}

Captura de pantalla del aula virtual: Zona de progreso - Unidad 2 y Zona de clausura

\section{Tema 2}

UNIDAD 2

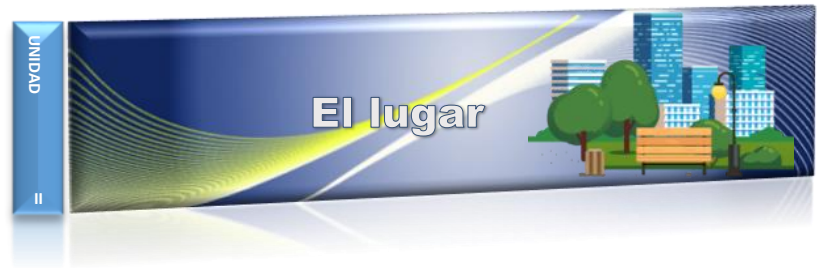

Fase de formulación e investigación mediante la actitud de búsqueda y exploración para el conocimiento del problema

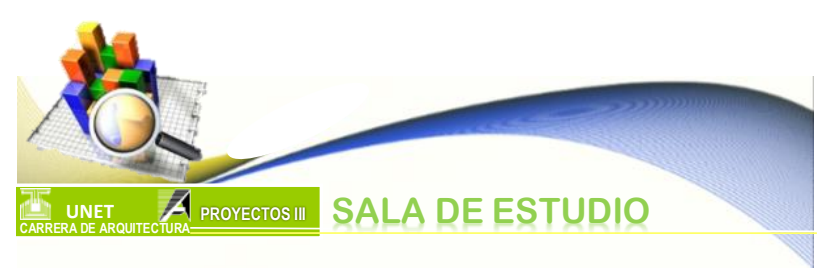

Preliminares: Organizador Previo

Objeto de aprendizaje Unidad II

ACTIVIDADES QUE REQUIEREN ATENCIÓN

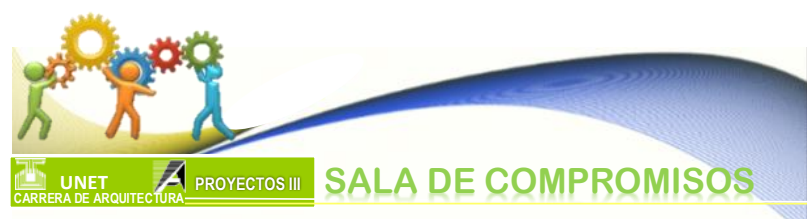

Tarea 3. Informe

Tarea 4. Cuadro Sinópico

Tarea 5. Fichas Técnicas

\section{Tema 8}

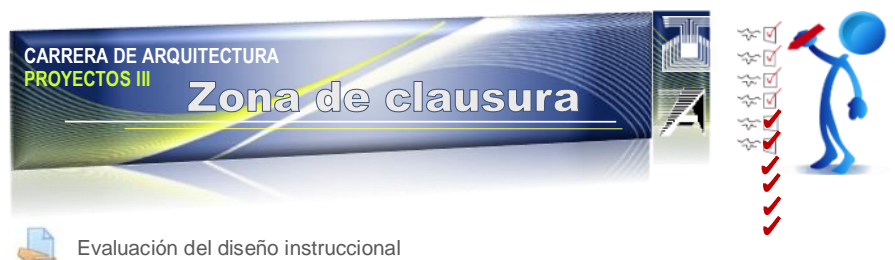

Evaluación del diseño instruccional

Evaluación de la calidad docente 


\subsubsection{Evaluación}

Durante la realización del DI, se revisó cada etapa del modelo (auto-reflexión), se planteó la evaluación de calidad del diseño instruccional, de los cinco Objetos de Aprendizaje, y del aula virtual con el uso de escalas. En la fase de implementación se prevé la retroalimentación mediante la observación directa, con el uso de escalas y analíticas de aprendizaje resultantes de la participación del docente y del estudiante.

La evaluación de calidad del DI antes de la implementación del aula, empleó 5 expertos (en diseño instruccional, evaluación y currículo) utilizó el Inventario del DI en cursos de formación de Coronado y Barraza (2018) estructurado en las dimensiones: claridad de la información (6 ítems), práctica reflexiva ( 6 ítems), realimentación informativa (6 ítems) y motivación intrínseca y extrínseca (7 ítems). La medición corresponde a un escalamiento de Likert con los valores: 1 (Nada), 2 (Casi nada), 3 (Casi siempre) y 4 (Siempre).

En la dimensión claridad de la información el 93\% promedio la valoró con 4 (Siempre) y el 7\% restante con 3 (Casi siempre). La distribución de los resultados indica que el 100\% asignó 4 (Siempre) a los indicadores: se describen los objetivos del curso, se presentan los aprendizajes esperados, se puntualiza la finalidad de las actividades y las formas de evaluación. El 80\% calificó con 4 (Siempre) y el 20\% con 3 (Casi siempre) a los estimadores: se ejemplifican los conceptos complejos y se ofrecen ejemplos variados.

En la dimensión promueve la práctica reflexiva el 90\% promedio designó 4 (Siempre) y el 10\% restante 3 (Casi siempre). La repartición de los resultados señala que el 100\% valoró con 4 (Siempre) a los indicadores: se activa la participación y se vinculan con las actividades, se promueve el análisis de las ideas extraídas de las participaciones, y se propone la exploración de soluciones a diversos problemas. El 80\% evaluó con 4 (Siempre) y el 20\% con 3 (Casi siempre) al estimador: se fomenta la reflexión en torno a las participaciones. Y el $60 \%$ calificó con 4 (Siempre) y el $40 \%$ con 3 (Casi siempre) al indicador: se propone la confrontación de situaciones concretas.

Con respecto a la dimensión realimentación informativa, el 73\% promedio la estimó con 4 (Siempre) y el $27 \%$ restante con 3 (Casi siempre). La asignación de estos valores señala que el 100\% calificó con 4 (Siempre) a los estimadores: se ofrecen instrucciones claras, se orienta de forma sencilla, se contemplan las características de los participantes y se muestra dominio del tema por parte del asesor. El 100\% valoró con 3 (Casi siempre) al estimador: se ofrecen referencias vigentes. El 60\% asignó 4 (Siempre) y el 40\% 3 (Casi siempre) al indicador: se reafirman las explicaciones cuando es necesario.

En la dimensión fuerte motivación intrínseca y extrínseca, el 100\% la evaluó con 4 (Siempre) los 7 indicadores: realiza una sistematización de las actividades, exhibe una metodología en las actividades, favorece la generación de ambientes de aprendizaje, se plantean actividades motivadoras y se relacionan con los objetivos planteados, se re-orienta el trabajo de algunas actividades para lograr los objetivos, y se activan los conocimientos previos. 


\section{DISCUSIÓN Y CONCLUSIONES}

El perfil obtenido de los estudiantes fue: A) Estilos de aprendizaje, tipo de información a percibir, Sensitivo (63\%) Intuitivo (37\%); b) Vía sensorial para captar la información, Visual (97\%) Verbal (3\%); c) forma de adquirir la información, Activo (71\%) Reflexivo (29\%); d) Modo para el entendimiento de contenidos, Secuencial (66\%) Global (34\%); B) Nivel de habilidades y destrezas digitales, alto $(78,70 \%)$, medio $(18,00 \%)$ y bajo $(3,30 \%)$; y C) Competencias y habilidades en el proceso de diseño arquitectónico, conceptuales $(80,2 \%)$ y procedimentales (24\%). En el diseño instruccional el perfil obtenido influyó en los aspectos pedagógicos, de contenido y tecnológicos, materializando las estrategias, los recursos y medios didácticos que atiendan la diversidad y estilos preferentes de aprendizaje de los estudiantes, para desarrollar adecuadamente los nuevos conocimientos a construir posibilitando el saber, el saber hacer y el saber estar en los procesos de formación de la actividad proyectual de un arquitecto. Situación que determinó el diseño de cinco Objetos de Aprendizaje basados en el método de la WebQuest para las cinco primeras unidades didácticas y tres videos de contenido.

El diseño del aula, la definición de conocimientos (conceptuales, procedimentales y actitudinales) y de la estrategia instruccional permitió la propuesta de 21 actividades de aprendizaje, las cuales se les definió el medio tecnológico a utilizar, la selección de recursos y los modos de evaluación. La instrucción se adecuó al pensum de la carrera de arquitectura de la Universidad Nacional Experimental del Táchira (2007) aplicado a Proyectos III con una duración de 16 semanas, se estructuró en siete unidades didácticas fundamentadas en las fases del Proceso de Diseño de Cantú (1998): formulación del problema, el lugar, programación de áreas, diagramas de relaciones, zonificación, síntesis conceptual y diseño.

El aula virtual diseñada se basó en los principios de Merril y los modelos de Diseño Instruccional: ADDIE, y Dick y Carey. Su adaptación en el aprendizaje del proceso del diseño arquitectónico orientó la propuesta de situaciones de enseñanza bajo la instrucción centrada en tareas y la metodología del aprendizaje basado en problemas para el entrenamiento de competencias genéricas y específicas, el pensamiento crítico y el aprendizaje autorregulado, la cual favoreció un ambiente de aprendizaje con calidad en cuanto a: claridad de la información (93\%), práctica reflexiva (90\%), realimentación informativa (73\%) y motivación (100\%).

\section{REFERENCIAS}

Aguilar, R. (2004). La Guía Didáctica, un material educativo para promover el aprendizaje autónomo. Evaluación y mejoramiento de su calidad en la modalidad abierta y a distancia de la UTPL. Revista Iberoamericana de Educación a Distancia, 7(1-2), 179-192. https://doi.org/10.5944/ried.7.1-2.1082

Alkiser, Y. (2017). Blended Learning: Architectural Design Studio Experiences Using Housing in Istanbul. Journal of Problem Based Learning in Higher Education, 5(1), 126-137. http://dx.doi.org/10.5278/ojs.jpblhe.v0i0.1553 
Avendaño, W. y Parada-Trujillo, A. (2012). El mapa cognitivo en los procesos de evaluación del aprendizaje. Investigación \& Desarrollo, 20(2), 334-365. http://www.redalyc.org/articulo.oa?id=26824854005

Bashier, F. (2014). Reflections on architectural design education: The return of rationalism in the studio. Frontiers of Architectural Research, 3(4), 424-430. https://doi.org/10.1016/i.foar.2014.08.004

Candela, B. (2016). La ciencia del diseño educativo. Programa Editorial de la Universidad del valle.

Cantú, I. (1998). Una aportación metodológica para desarrollar la creatividad en el diseño. Modelo para la conceptualización del diseño arquitectónico. [Tesis de maestría no publicada]. Universidad Autónoma de Nuevo León.

Coordinación de Educación a Distancia. (2016). Instructivo para el desarrollo de un aula virtual. Universidad del Táchira - Consejo Académico Resolución № CAE 003. https://drive.google.com/file/d/OB86cA2IYiyRSeTZnbDdPZDAOSkE/view? usp=sharing

Coronado, J. y Barraza, A. (2018). Estudio instrumental para verificar el diseño instruccional en cursos de formación. Diálogos Pedagógicos, XVI(31), 20-34. https://doi.org/10.22529/dp.2018.16(31)02

Dick, W., Carey, L. y Carey, J. (2015). The sistematic design of instruction (8va ed.). Pearson Education.

Dorrego, E. (1994). Modelo para la producción y evaluación formativa de medios instruccionales, aplicado al video y al software. Informática Educativa. $2^{\circ}$ Congreso Ibero-americano de Educación en Informática, Portugal. http://www.ufrgs.br/niee/eventos/RIBIE/1994/index.php

Felder, R. y Soloman, B. (1997, mayo 15). Index of Learning Styles (ILS ${ }^{\circledR}$ ) Questionnaire. NC State University. https://www.webtools.ncsu.edu/learningstyles/

Fleischmann, K. (2018). Hype or help? Technology-enhanced learning in the design classroom: an experiment in online design collaboration. International Journal of Arts \& Sciences. 11(1), 331-342. http://www.universitypublications.net/ijas/1101/html/M8K144.xml

Fleischmann, K. (2020). Online design education: Searching for a middle ground. Arts and Humanities in Higher Education, 19(1), 36-57. https://doi.org/10.1177/1474022218758231

García-Ros, R. (2011). Análisis y validación de una rúbrica para evaluar habilidades de presentación oral en contextos universitarios. Electronic Journal of Research in Educational

Psychology,

9(3), 1043-1062. www.redalyc.org/articulo.oa?id=293122852004 
Guevara, O. (2013). Análisis del proceso de enseñanza aprendizaje de la disciplina proyecto arquitectónico, en la carrera de arquitectura, en el contexto del aula (Publicación No. 9788449036699) [Tesis doctoral, Universidad Autónoma de Barcelona]. Repositorio Campus UAB. https://www.tdx.cat/handle/10803/116191

Guzmán, Y., Flores, R. y Tirado, F. (2012). La evaluación de la competencia argumentativa en foros de discusión en línea a través de rúbricas. Innovación Educativa, 12(60), 17-40. http://www.scielo.org.mx/scielo.php?script=sci_arttext\&pid=S166526732012000300003\&lng=es\&tlng=es.

Hill, A. (2017). The 'Tutorless' Design Studio: A Radical Experiment in Blended Learning. Journal of Problem Based Learning in Higher Education, 5(1), 111-125. http://dx.doi.org/10.5278/ojs.jpblhe.v0i0.1550

Joklová, V. y Pifko, H. (2015). Innovation in architectural education - OIKONET experience.

Global Journal of Engineering Education, 17(3), 124-131. http://www.wiete.com.au/journals/GJEE/Publish/vol17no3/04-Joklova-Pifko.pdf

Khalil, M. y Elkhider, I. (2016). Applying learning theories and instructional design models for effective instruction. Advances in Physiology Education, 40(2), 147-156. https://doi.org/10.1152/advan.00138.2015

Kolb, D. (2015). Expential learning as the source of learning and development ( $2^{\mathrm{a}}$ ed.). PrenticeHall.

López, K. y Peña, (2019). Escribir para convencer: experiencia de diseño instruccional en contextos digitales de autoaprendizaje. Apertura, 12(1), 22-38. http://dx.doi.org/10.32870/Ap.v12n1.1807

Lotz, N., Jones, D. y Holden, G. (2015). Social engagement in online design pedagogies. Proceedings of the 3rd International Conference for Design Education Researchers, Finlandia. 1645-1668. http://oro.open.ac.uk/43592/

Masdéu, M. y Fuses, J. (2017). Reconceptualizing the Design studio in architectural education: distance learning and blended learning as transformation factors. International Journal of Architectural Research, 11(2), 6-23.

Mattingly, A. (2011). Mind and method: an examination of cognitive activities in the design process [Tesis doctoral, Universidad Estatal de Colorado]. Repositorio campus CSU. http://hdl.handle.net/10217/47431

Merril D. (2012). First Principles of Instruction: Identifying and Designing Effective, Efficient, and Engaging Instruction. Pfeiffer (John Wiley \& Sons).

Molina-Zambrano, M. (2018). Aula virtual para el aprendizaje del proceso de diseño arquitectónico [Tesis de maestría no publicada]. Universidad Nacional Experimental del Táchira. 
Molina-Zambrano, M. y Ruiz-Morales, Y. A. (2020). Diseño de Objeto de Aprendizaje basado en una WebQuest para la programación de áreas que definen el espacio arquitectónico. Edutec. Revista Electrónica De Tecnología Educativa, (74), 127-148. https://doi.org/10.21556/edutec.2020.74.1623

Mora, G., Riquelme, L., Troncoso, J. y Escobar, B. (2018). Validación de una Matriz para Evaluar Monografías en Estudiantes Universitarios. Formación universitaria, 11(1), 63-76. http://dx.doi.org/10.4067/S0718-50062018000100063

Oman, S., Tumer, I., Wood, K. y Seepersad, C. (2013). A comparison of creativity and innovation metrics and sample validation through in-class design projects. Research in Engineering Design, 24, 65-92. https://doi.org/10.1007/s00163-012-0138-9

Opoko, P., Adeokun, C. y Dare, O. (2015). Innovations in the teaching of architectural studio: The covenant experience. Global Journal on Humanites \& Social Sciences, Croacia, 1, $73-$ 79. http://eprints.covenantuniversity.edu.ng/id/eprint/3642

Organista, J., Lavigne, G., Serrano, A. y Sandoval, M. (2017). Desarrollo de un cuestionario para estimar las habilidades digitales de estudiantes universitarios. Revista Complutense de Educación, 28(1), 325-343. http://dx.doi.org/10.5209/rev RCED.2017.v28.n1.49802

Prado-Rodríguez, A. (2021). Conectivismo y diseño instruccional: ecología de aprendizajes para la universidad del siglo XXI en México. Márgenes, Revista de Educación de la Universidad de Málaga, 2(1), 4-20. https://doi.org/10.24310/mgnmar.v2i1.9349

Reiter, R., Illies, M., Kobe, L., Buboltz, C. y Nimps, T. (2009). Creativity and domain specificity: The effect of task Type on Multiple Indexes of Creative Problem Solving. Psychology of Aesthetics, Creativity, and the Arts, 3(2), 73-80. https://doi.org/10.1037/a0013410

Reigeluth, Ch. (2016). Teoría instruccional y tecnología para el nuevo paradigma de la educación. Revista de Educación a Distancia, 50, 1-20. https://www.um.es/ead/red/50/reigeluth esp.pdf

Ruiz-Morales, Y., García-García, M., Biencinto-López, C. y Carpintero, E. (2017). Evaluación de competencias genéricas en el ámbito universitario a través de entornos virtuales: Una revisión narrativa. RELIEVE, 23(1), 1-15. http://doi.org/10.7203/relieve.23.1.7183

Salama, A. (2015). Spatial Design Education: New Directions for Pedagogy in Architecture and Beyond. Routledge.

Schön, D. (1983). The reflective practitioner: How professionals think in action. Basic Books.

Tur, G. y Urbina, S. (2016). Rúbrica para la evaluación de portafolios electrónicos en el entorno de la web social. Revista De Medios Y Educación, (48), 83-96. https://doi.org/10.12795/pixelbit.2016.i48.06

Universidad Nacional Experimental del Táchira (UNET). (2007). Carrera de Arquitectura: Reforma Curricular 2007 [Libro blanco]. Departamento de Arquitectura. 
Vélez Amador, R. (2017). Modelo de producción de vídeos didácticos para la modalidad presencial de la enseñanza universitaria. Revista de comunicación de la SEECI, (43) 6997. https://doi.org/10.15198/seeci.2017.43.69-97

Webster, H. (2004). Facilitating critically reflective learning: excavating the role of the design tutor in architectural education. Art, Design \& Communication in Higher Education, 2(3), 101-111. https://doi.org/10.1386/adch.2.3.101/0

\section{Para citar este artículo:}

Molina Zambrano, M., y Ruiz Morales, Y. A. (2021). Aula virtual para el aprendizaje del proceso de diseño arquitectónico. Edutec. Revista Electrónica De Tecnología Educativa, (78), 264-283. https://doi.org/10.21556/edutec.2021.78.2139 


\section{ANEXOS}

Resultados de la evaluación formativa del Diseño Instruccional

Tabla 1

Dimensión claridad de la información

\begin{tabular}{|c|c|c|c|c|c|c|c|c|c|}
\hline \multirow{2}{*}{ Ítem } & \multirow{2}{*}{ Descripción } & \multicolumn{2}{|c|}{1} & \multicolumn{2}{|c|}{2} & \multicolumn{2}{|c|}{3} & \multicolumn{2}{|c|}{4} \\
\hline & & $\mathrm{fi}$ & $\% \mathrm{fi}$ & $\mathrm{fi}$ & $\% \mathrm{fi}$ & $\mathrm{fi}$ & $\% \mathrm{fi}$ & $\mathrm{fi}$ & $\% \mathrm{fi}$ \\
\hline 1 & Se describen los objetivos del curso & 0 & 0 & 0 & 0 & 0 & 0 & 5 & 100 \\
\hline 2 & Se puntualiza la finalidad de las actividades & 0 & 0 & 0 & 0 & 0 & 0 & 5 & 100 \\
\hline 3 & Se ejemplifican conceptos complejos & 0 & 0 & 0 & 0 & 1 & 20 & 4 & 80 \\
\hline 4 & Se demuestran ejemplos variados & 0 & 0 & 0 & 0 & 1 & 20 & 4 & 80 \\
\hline 5 & Se presentan los aprendizajes esperados & 0 & 0 & 0 & 0 & 0 & 0 & 5 & 100 \\
\hline 6 & Se puntualizan las formas de evaluación & 0 & 0 & 0 & 0 & 0 & 0 & 5 & 100 \\
\hline & Promedio/media & & & & & & 7 & & 93 \\
\hline
\end{tabular}

Tabla 2

Dimensión promueve la práctica reflexiva

\begin{tabular}{|c|c|c|c|c|c|c|c|c|c|}
\hline \multirow{2}{*}{ Ítem } & \multirow{2}{*}{ Descripción } & \multicolumn{2}{|c|}{1} & \multicolumn{2}{|c|}{2} & \multicolumn{2}{|c|}{3} & \multicolumn{2}{|c|}{4} \\
\hline & & $\mathrm{fi}$ & $\% \mathrm{fi}$ & $\mathrm{fi}$ & $\% \mathrm{fi}$ & $\mathrm{fi}$ & $\% \mathrm{fi}$ & $\mathrm{fi}$ & $\% \mathrm{fi}$ \\
\hline 7 & Se activa la participación & 0 & 0 & 0 & 0 & 0 & 0 & 5 & 100 \\
\hline 8 & Se vincula las participaciones con las actividades & 0 & 0 & 0 & 0 & 0 & 0 & 5 & 100 \\
\hline 9 & $\begin{array}{l}\text { Se fomenta la reflexión en torno a las } \\
\text { participaciones }\end{array}$ & 0 & 0 & 0 & 0 & 1 & 20 & 4 & 80 \\
\hline 10 & $\begin{array}{l}\text { Se promueve el análisis de las ideas extraídas de las } \\
\text { participaciones }\end{array}$ & 0 & 0 & 0 & 0 & 0 & 0 & 5 & 100 \\
\hline 11 & $\begin{array}{l}\text { Se propone la confrontación de situaciones } \\
\text { concretas }\end{array}$ & 0 & 0 & 0 & 0 & 2 & 40 & 3 & 60 \\
\hline 12 & $\begin{array}{l}\text { Se propone la exploración de soluciones a diversos } \\
\text { problemas }\end{array}$ & 0 & 0 & 0 & 0 & 0 & 0 & 5 & 100 \\
\hline & Promedio/media & & & & & & 10 & & 90 \\
\hline
\end{tabular}

Tabla 3

Dimensión realimentación informativa

\begin{tabular}{clccccccccc}
\hline \multirow{2}{*}{ Ítem Descripción } & \multicolumn{1}{c}{1} & \multicolumn{2}{c}{2} & \multicolumn{3}{c}{4} \\
\cline { 3 - 9 } & & $\mathrm{fi}$ & $\% \mathrm{fi}$ & $\mathrm{fi}$ & $\% \mathrm{fi}$ & $\mathrm{fi}$ & $\% \mathrm{fi}$ & $\mathrm{fi}$ & $\% \mathrm{fi}$ \\
\hline 13 & Se ofrece instrucciones claras & 0 & 0 & 0 & 0 & 0 & 5 & 100 \\
14 & Se orienta de forma sencilla & 0 & 0 & 0 & 0 & 0 & 0 & 5 & 100 \\
15 & Se contemplan las características de los & 0 & 0 & 0 & 0 & 0 & 0 & 5 & 100 \\
16 & participantes & Se reafirman las explicaciones cuando es necesario & 0 & 0 & 0 & 0 & 3 & 60 & 2 & 40 \\
17 & Se muestra dominio del tema por parte del asesor & 0 & 0 & 0 & 0 & 0 & 0 & 5 & 100 \\
18 & Se ofrecen referencias vigentes & 0 & 0 & 0 & 0 & 5 & 100 & 0 & 0 \\
\hline & Promedio/media & & & & & & & & & \\
\hline
\end{tabular}




\section{Tabla 4}

Dimensión fuerte motivación intrínseca

\begin{tabular}{|c|c|c|c|c|c|c|c|c|c|}
\hline \multirow{2}{*}{ Ítem } & \multirow{2}{*}{ Descripción } & \multicolumn{2}{|c|}{1} & \multicolumn{2}{|c|}{2} & \multicolumn{2}{|c|}{3} & \multicolumn{2}{|c|}{4} \\
\hline & & $\mathrm{fi}$ & $\% \mathrm{fi}$ & $\mathrm{fi}$ & $\% \mathrm{fi}$ & $\mathrm{fi}$ & $\% \mathrm{fi}$ & $\mathrm{fi}$ & $\% \mathrm{fi}$ \\
\hline 19 & Se realiza una sistematización de las actividades & 0 & 0 & 0 & 0 & 0 & 0 & 5 & 100 \\
\hline 20 & Se exhibe una metodología en las actividades & 0 & 0 & 0 & 0 & 0 & 0 & 5 & 100 \\
\hline 21 & $\begin{array}{l}\text { Se favorece la generación de ambientes de } \\
\text { aprendizaje }\end{array}$ & 0 & 0 & 0 & 0 & 0 & 0 & 5 & 100 \\
\hline 22 & Se plantean actividades motivantes & 0 & 0 & 0 & 0 & 0 & 0 & 5 & 100 \\
\hline 23 & $\begin{array}{l}\text { Se relacionan las actividades con los objetivos } \\
\text { planteados }\end{array}$ & 0 & 0 & 0 & 0 & 0 & 0 & 5 & 100 \\
\hline 24 & $\begin{array}{l}\text { Se reorienta el trabajo de algunas actividades para } \\
\text { lograr los objetivos }\end{array}$ & 0 & 0 & 0 & 0 & 0 & 0 & 5 & 100 \\
\hline \multirow[t]{2}{*}{25} & Se activan los conocimientos previos & 0 & 0 & 0 & 0 & 0 & 0 & 5 & 100 \\
\hline & Promedio/media & & & & & & 0 & & 100 \\
\hline
\end{tabular}

